\title{
Tecnologías de la información y comunicación en estudiantes de Educación Básica de la ciudad de Osorno
}

\author{
Yorka Tatiana Ortiz Ruiz \\ Magister en Informática Educativa \\ Académico Línea Informática Educativa \\ Universidad de Los Lagos, Chile \\ Email: yortiz@ulagos.cl \\ Recepción: 28-09-2015 / Aceptación: 10-11-2015
}

Resumen

Actualmente, las tecnologías se vinculan en todos los ámbitos de la sociedad y son las nuevas generaciones las más familiarizadas en su manejo técnico; son los jóvenes de ahora denominados «nativos digitales». Por medio de esta investigación de tipo cuantitativo se buscó caracterizar el uso de los recursos tecnológicos - que tienen a su disposición - tanto en el establecimiento educacional como en su hogar, los estudiantes de quinto y sexto año de Educación Básica de la Escuela España de la ciudad de Osorno en Chile. Este estudio sirve para aumentar el conocimiento que los docentes deben tener de sus estudiantes, y así poder orientar su accionar con base en las destrezas y habilidades que estos tengan.

Entre los resultados destaca que, pese a que los estudiantes investigados forman parte de familias catalogadas en un nivel «vulnerable», cuentan con acceso a diversas tecnologías como televisores, teléfonos y computadores portátiles con acceso a internet en sus hogares. Esto insumos se ocupan principalmente en actividades de entretención, por el uso mayoritario que dan a espacios de la denominada web 2.0, tales como la red social Facebook y el portal de videos Youtube. También se descubrió que existe una gran variedad de recursos tecnológicos en el establecimiento educacional, los cuales son utilizados por el docente y los estudiantes. Estos recursos son los más tradicionales: computadores, impresora y proyector multimedia, dejando de lado cámaras y pizarras digitales.

Palabras clave: Tecnologías de la información y comunicación; educación; nativos digitales, recursos educativos

\begin{abstract}
Technologies are currently linked in all areas of society and the younger generation are most familiar in its technical management; young people are now called "digital natives". This quantitative research was aimed at characterizing the use of technological resources - they have at their disposal - both in the educational establishment at home, students in fifth and sixth year Basic Education School Spain of city of Osorno in Chile. This study serves to increase awareness that teachers should have of their students, so they can guide their actions based on the skills and abilities that they have.

The results highlighted that, although the students surveyed are part of families cataloged in a 'vulnerable ' level, they have access to various technologies such as televisions, phones and laptop computers with internet access in their homes. These are mainly engaged in entertainment activities, the majority use spaces that give web 2.0, such as social network Facebook and video portal YouTube. It was also discovered that there are a variety of technological resources in the educational establishment, which are used by teachers and students. These resources are the most traditional: computers, printers and multimedia projector, leaving aside cameras and whiteboards .
\end{abstract}

Key Words: Information and Communication Technologies, Education, digital natives, educational resources 


\section{Introducción}

Existe una sociedad en constante cambio, a la cual algunos autores como Castells (2001) denominan Sociedad de la Información o Sociedad del Conocimiento; es decir, una sociedad que se caracteriza por el cúmulo de información a su alcance y cuyo interés es poder administrar en forma eficiente dicha información, lo que se traduce en poder analizar, sintetizar y tomar decisiones acertadas considerando el manejo de dicha información.

Según la UNESCO (2013), al referirse a las Tecnologías

Las Tecnologías de la Información y la Comunicación (TIC) han tenido un desarrollo explosivo en la última parte del siglo XX y el comienzo del siglo XXI, al punto de que han dado forma a lo que se denomina «Sociedad del Conocimiento» o «de la Información». Prácticamente no hay un solo ámbito de la vida humana que no se haya visto impactada por este desarrollo: la salud, las finanzas, los mercados laborales, las comunicaciones, el gobierno, la productividad industrial, etc. El conocimiento se multiplica más rápido que nunca antes y se distribuye de manera prácticamente instantánea.

Por lo anterior, existe en la actualidad una brecha importante en torno a las destrezas y habilidades para el manejo de recursos tecnológicos presentes en profesores y estudiantes. Es lo que se denomina «brecha digital», que algunos autores como Serrano y Martínez (2003) definen como: la separación que existe entre las personas (comunidades, estados, países) que utilizan las Tecnologías de Información y Comunicación como una parte rutinaria de su vida diaria y aquellas que no tienen acceso a las mismas y que aunque las tengan no saben cómo utilizarlas.

Es una realidad que los adultos manejan las computadoras, comprenden la telefonía móvil; sin embargo, proceden de otra generación: una generación más metódica, una generación que necesita analizar más las cosas; una generación que procede de un cambio educacional, pasando desde el conductismo hacia el constructivismo. Esto es lo que Marc Prensky (2010) denomina nativos e inmigrantes digitales.

La generación de hoy ya no es igual a la de diez años atrás, está presente en las escuelas, en las familias, en el diario vivir. Los niños y jóvenes de hoy están insertos en un mundo tecnológico que muchas veces se les escapa de las manos.

El Ministerio de Educación de Chile (MINEDUC) considera de gran importancia el ingreso de las tecnologías de la información y la comunicación en las aulas, debido a esto surge el proyecto ENLACES el cual

Nació en 1992 como un proyecto piloto, con el objetivo de entregar infraestructura tecnológica, contenidos digitales educativos y capacitación docente en todas las escuelas y liceos subvencionados del país. A lo largo de estos años Enlaces se ha transformado en el motor de la innovación y la incorporación de las Nuevas Tecnologías 
de la Información y Comunicación en la educación chilena (MINEDUC, s.f.).

Esta investigación tuvo como propósito caracterizar el uso de los recursos tecnológicos que tienen disponibles los estudiantes de quinto $\mathrm{y}$ sexto año de Educación Básica de la Escuela España de la ciudad de Osorno en Chile.

La Escuela España, y muchos otros establecimientos, presentan laboratorios de computación con toda la implementación necesaria; esto con el objetivo establecido por el Ministerio de Educación chileno de que los estudiantes desarrollen diversas habilidades TIC para el aprendizaje, las que se definen como «La capacidad de resolver problemas de información, comunicación y conocimiento así como dilemas legales, sociales y éticos en ambiente digital». ${ }^{1}$

Por tal razón, mediante este estudio se indagó sobre el uso que hacen los estudiantes con respecto a los recursos tecnológicos que tiene el establecimiento. Es importante mencionar que los estudiantes de hoy están invadidos por estas nuevas tecnologías y las consideran parte de su entorno, ya que están presentes desde su nacimiento, y por ende, les son atractivas desde pequeños. Alvin Toffler (1993) plantea una teoría donde existen tres hitos en la historia de la humanidad a los que él llama «olas».

- La primera la «agraria»,

- La segunda la «industrial»,

- la tercera la «ola de la información».
Es esta última la que está avanzando a pasos agigantados, y se puede apreciar como los niños se aproximan a pantallas computarizadas con el mismo o mayor interés que si estuviesen en el mundo real; las relaciones personales se afianzan a través de chat, conversaciones por páginas sociales, telefonía celular y videojuegos, sean estos online o mediante un dispositivo (Wii, Xbox).

\section{Metodología}

El estudio se enmarca en un paradigma explicativo, no experimental y cuantitativo (Hernández, Fernández y Baptista, 2010); en el convencimiento de lograr antecedentes lo más objetivos posibles, se utilizó la técnica de la encuesta para la recogida y el análisis de datos.

La población estuvo formada por los estudiantes de quinto y sexto año de Educación Básica de la Escuela España de la ciudad de Osorno, Chile, específicamente quienes cursaron estudios en el segundo semestre del año 2013. En total fueron 55 estudiantes, conformándose un 19.1\% de la población del establecimiento.

La encuesta fue aplicada a un grupo piloto integrado por cinco estudiantes de sexto básico y cuatro estudiantes de quinto básico, escogidos al azar. Este grupo denominado «piloto» permitió enfrentar el instrumento a un grupo reducido de estudiantes, para que ellos pudieran entregar su opinión en torno a la claridad de las preguntas, el lenguaje de las mismas y una adecuada comprensión de acuerdo a su edad.

1. Extraído de Habilidades TIC en estudiantes, disponible en: http: //www.enlaces.cl/index.php? $\mathrm{t}=44 \& \mathrm{i}=2 \& \mathrm{cc}=2285 \& \mathrm{tm}=2$ 
El instrumento de recogida de información utilizado fue la encuesta semiestructurada, definida según Hernández, Fernández y Sampieri (2010) como «el método de investigación capaz de dar respuestas a problemas tanto en términos descriptivos como de relación de variables, tras la recogida de información sistemática, según un diseño previamente establecido que asegure el rigor de la información obtenida». De esta forma, este instrumento puede ser utilizado en forma segura para entregar descripciones precisas de los objetos o variables en estudio; además de permitir detectar patrones y relaciones entre las características descritas, y establecer relaciones entre los diferentes eventos o situaciones expuestas.

El instrumento estuvo compuesto por tres ítems más un espacio introductorio sobre aspectos generales e información de identificación del estudiante. El ítem uno trató sobre conocimiento y uso de recursos tecnológicos en el establecimiento educacional; ítem dos, sobre recursos que pertenecientes al estudiante; el ítem tres, sobre los usos que él da a los recursos de los que dispone, con diferentes tipologías y con respuestas cerradas.

El proceso de elaboración de las encuestas señaladas se realizó dentro de las siguientes etapas: a) revisión de literatura; b) revisión de otros instrumentos elaborados en investigaciones referidas a la utilización o incorporación de recursos tecnológicos en diferentes contextos, como por ejemplo: Antón (2010); Cebrián de la Serna (2005); Tobón, (2009); c) elaboración de la primera versión de los ins- trumentos d) validación con juicio de expertos e) estudio piloto.

\section{Resultados}

Con respecto a los antecedentes, los estudiantes debieron seleccionar en su encuesta el género, su edad y la disponibilidad de acceso a internet en su hogar.

Tabla 1. Distribución de género

\begin{tabular}{|c|c|}
\hline Genero & Porcentaje \\
\hline Femenino & $53 \%$ \\
\hline Masculino & $47 \%$ \\
\hline
\end{tabular}

Con los datos recolectados se pudo evidenciar que el 53\% de los encuestados fue de género femenino y el $47 \%$ restante fue de género masculino.

Tabla 2. Distribución por edad

\begin{tabular}{|c|c|}
\hline Edad (años) & Porcentaje \\
\hline 10 & $29 \%$ \\
\hline 11 & $35 \%$ \\
\hline 12 & $24 \%$ \\
\hline 13 & $7 \%$ \\
\hline 14 & $5 \%$ \\
\hline
\end{tabular}

El cien por ciento de las edades de los encuestado fluctuó, mayoritariamente, entre los 10 a 12 años; correspondiendo esto a un $88 \%$ de la población encuestada. El $12 \%$ restante se encontró en un rango de edad mayor al promedio (13 a 14 años). 


\section{Disponibilidad}

Tabla 3. Disponibilidad de internet en el hogar

\begin{tabular}{|c|c|c|}
\cline { 2 - 3 } \multicolumn{1}{c|}{} & Sí & No \\
\hline $\begin{array}{c}\text { ¿Dispones de } \\
\text { internet en } \\
\text { tu hogar? }\end{array}$ & $55 \%$ & \\
\hline
\end{tabular}

En la tabla 3 se puede observar que, ante la pregunta ${ }_{i}$ Dispones de internet en tu hogar?, los encuestados respondieron mayoritariamente de manera afirmativa, obtenido un $55 \%$ de aprobación, correspondiente a 30 estudiantes distribuidos entre quinto y sexto año básico.

Este resultado llama la atención por el gran porcentaje de estudiantes que cuentan con este servicio, ya que se está frente a un establecimiento clasificado como vulnerable, de acuerdo al Reglamento sobre obligatoriedad de establecimientos educacionales, según el decreto 196 publicado el 09 de enero del año $2006^{2}$ (Ministerio de Educación). Acorde a este documento, un establecimiento es catalogado de esta forma si cuenta con al menos un $15 \%$ de alumnos en condiciones de vulnerabilidad socioeconómica como requisito para impetrar la subvención, para lo cual se considerará el nivel socioeconómico de la familia, el nivel de escolaridad de los padres o apoderados y el entorno del establecimiento. resultados de diversos estudios, como el de Adolfo Oliva (2012) denominado «La brecha
Esta realidad se ve reflejada a través de los

digital en Chile: más allá de la red de acceso», que han concluido que las principales variables que inciden en la adopción de internet por banda ancha son el precio del servicio, el precio de los equipos, el nivel de ingreso y factores socio demográficos.

Además es importante destacar que las oportunidades de accesibilidad están presentes en los hogares chilenos, ya que se puede optar desde un modem de Banda Ancha Móvil hasta servicios más rápidos; esto se ve reflejado en el estudio realizado por Richard Buchi ${ }^{3}$ (2002) quién desarrolló el estudio de Internet en Chile. Mediante su investigación demostró que desde el tercer quintil, los chilenos contratan internet ya que reciben productos gratis que se ofrecen, aprovechando incorporarse sin un costo fijo - que es lo que más los asusta - ya que no quedan amarrados a un pago o suscripción y tienen la libertad de usarlo, pagarlo o salirse, si no les gusta el servicio.

Tabla 4. Recursos disponibles en el establecimiento

\begin{tabular}{|c|c|}
\hline Recursos & Porcentaje \\
\hline $\begin{array}{c}\text { Pizarras } \\
\text { digitales }\end{array}$ & $0 \%$ \\
\hline $\begin{array}{c}\text { Videograba- } \\
\text { doras }\end{array}$ & $16 \%$ \\
\hline Cámaras & $16 \%$ \\
\hline $\begin{array}{c}\text { Minicompo- } \\
\text { nente }\end{array}$ & $44 \%$ \\
\hline Notebook & $65 \%$ \\
\hline Netbook & $76 \%$ \\
\hline $\begin{array}{c}\text { Computador } \\
\text { de escritorio }\end{array}$ & $91 \%$ \\
\hline
\end{tabular}

2. Decreto 196. Disponible en: http://www.comunidadescolar.cl/marco_legal/Decretos/Decreto\%20196\%20Vulnerabilidad.pdf 3. Richard Buchi: es ingeniero civil titulado en la Universidad de Chile y MBA Wharton School of Business de la Universidad de Pennsylvania, EE.UU. Actual Gerente General de Entel Chile S.A., es Presidente de Entel Telefonía Personal y de Entelphone. 


\begin{tabular}{|c|c|}
\hline Recursos & Porcentaje \\
\hline Impresora & $95 \%$ \\
\hline Data & $98 \%$ \\
\hline $\begin{array}{c}\text { Fotocopia- } \\
\text { dora }\end{array}$ & $95 \%$ \\
\hline Otros & $4 \%$ \\
\hline
\end{tabular}

En la tabla 4 se muestran los resultados cuando a los estudiantes se les solicitó reconocer los recursos tecnológicos que existen en el establecimiento. Casi la totalidad aseguró que existe fotocopiadora y proyector multimedia; esto está asociado a que los estudiantes constantemente utilizan estos recursos. Sin embargo, es importante señalar el nulo conocimiento acerca de otros recursos presentes en el establecimiento, como son las videograbadoras y cámaras digítales, las cuales solo presentan un $16 \%$ de conocimiento por parte de los estudiantes. Esto se relaciona directamente con el poco uso que los docentes hacen de ellos para realizar trabajos los requieran.

\section{Utilización de recursos}

Tabla 5. Usos que el estudiante da a los recursos del establecimiento

\begin{tabular}{|c|c|}
\hline Usos & Porcentaje \\
\hline $\begin{array}{c}\text { Realizar tra- } \\
\text { bajos en clases }\end{array}$ & $89 \%$ \\
\hline Estudiar & $67 \%$ \\
\hline Entretenerme & $49 \%$ \\
\hline
\end{tabular}

En la tabla se puede observar que la gran mayoría de los estudiantes relaciona la pregunta a los computadores de escritorio presentes en la escuela, ya que es el recurso más utilizado. El $89 \%$ de los estudiantes entre quinto y sexto año básico reconoce que utiliza estos recursos para realizar trabajos en clases.

\section{Frecuencia de uso}

Tabla 6. Frecuencia en el uso de los recursos tecnológicos presentes en tu establecimiento

\begin{tabular}{|c|c|}
\hline Frecuencia & Porcentaje \\
\hline Todos los días & $5 \%$ \\
\hline $\begin{array}{c}\text { Algunos días } \\
\text { de la semana }\end{array}$ & $55 \%$ \\
\hline $\begin{array}{c}\text { Algunas veces } \\
\text { al mes }\end{array}$ & $31 \%$ \\
\hline Casi nunca & $9 \%$ \\
\hline
\end{tabular}

Los estudiantes, al responder la pregunta relacionada con frecuencia en el uso de los recursos tecnológicos presentes en tu establecimiento, señalaron utilizar solo algunos días a la semana los recursos, asociando estos a los computadores de escritorio que son los que ellos utilizan con mayor cotidianeidad. Esta información no puede ser contrastada con registros establecidos desde la unidad educativa, considerando que no existe una organización o coordinación que supervise el uso y lleve control del tipo de uso.

Recursos propios del estudiante

Tabla 7. Recursos propios del estudiante

\begin{tabular}{|c|c|}
\hline $\begin{array}{c}\text { Recursos que tie- } \\
\text { ne el estudiante } \\
\text { Xbox }\end{array}$ & Porcentaje \\
\hline Iphone & $5 \%$ \\
\hline Ipad & $5 \%$ \\
\hline Nintendo & $9 \%$ \\
\hline Netbook & $20 \%$ \\
\hline
\end{tabular}




\begin{tabular}{|c|c|}
\hline $\begin{array}{c}\text { Recursos que tie- } \\
\text { ne el estudiante }\end{array}$ & Porcentaje \\
\hline Wii & $15 \%$ \\
\hline Otro celular & $27 \%$ \\
\hline Tablet & $22 \%$ \\
\hline Pendrive & $29 \%$ \\
\hline Play & $33 \%$ \\
\hline $\begin{array}{c}\text { Computador de } \\
\text { escritorio }\end{array}$ & $49 \%$ \\
\hline Mp3-Mp4 & $36 \%$ \\
\hline $\begin{array}{c}\text { Celular con sis- } \\
\text { tema android }\end{array}$ & $56 \%$ \\
\hline Notebook & $49 \%$ \\
\hline T.V. & $78 \%$ \\
\hline Radio & $84 \%$ \\
\hline
\end{tabular}

Cuando se consulta por los recursos tecnológicos que tiene el estudiante se logra detectar que la radio y el televisor siguen siendo los recursos más utilizados por las familias chilenas. La tabla muestra las preferencias ante las distintas opciones. Estudios relacionados indican que la radio es el medio informativo más extendido, el más ampliamente utilizado, el de más fácil comprensión y manejo, y el más rápido en la comunicación de noticias. ${ }^{4}$

La UNESCO, en su publicación denominada «Boletín Proyecto Principal de Educación en América Latina y el Caribe», señala que durante los últimos treinta años, el medio televisivo ha tenido una enorme y constante expansión en América Latina. El número de estaciones emisoras de TV se ha multiplica- do, pasando de 250 equipos en el año 1965, a 1540 de ellos en 1985; es decir, su número ha crecido seis veces. Además, el parque de aparatos receptores de TV ha pasado de 32 por cada mil habitantes en 1965, a 138 televisores por cada mil personas en 1985. Esta cifra significa que en el presente existe como promedio un televisor por cada siete latinoamericanos.

Según el Instituto de Investigación en Ciencias Sociales, ICSO-UDP (2009), el 98.1\% de los chilenos tiene hoy en día una televisión a color en su hogar. Hace cinco años esta cifra era de 93.6\%; señalando, además, que hoy no importa el nivel socioeconómico para la tenencia de este bien. Se trata de un elemento presente en todas las casas. ${ }^{5}$

Además existen artículos que revelan que

prácticamente todos los niños y jóvenes, de cualquier edad, tienen un aparato de televisión en color en su casa. Casi el 60\% de los hogares disponen de dos o más televisores, y prácticamente todos los hogares con niños tienen un vídeo/dvd. Este nivel tecnológico hace que muchos niños y jóvenes tengan su propio equipamiento en la habitación, incluida a veces la conexión a internet, lo que hace que los controles parentales, por ejemplo, del uso en tiempo y contenidos de la televisión o su navegación en la red, sean mucho menor a los que se producían anteriormente.

4. Extraído de: http: //dspace.ceu.es/bitstream/10637/2129/2/Resumen.pdf

5. La Relación de los chilenos con la televisión e Internet. Disponible en: http://www.encuesta.udp.cl/wp-content/ uploads/2010/09/relacion-chilenos-tv-internet.pdf 


\section{Adquisición de recursos tecnológicos}

Tabla 8. Adquisición de Recursos

Tecnológicos del estudiante

\begin{tabular}{|c|c|}
\hline Personas & Porcentaje \\
\hline Amigos & $5 \%$ \\
\hline Abuelos & $22 \%$ \\
\hline Yo & $15 \%$ \\
\hline Otros familiares & $40 \%$ \\
\hline Papá & $60 \%$ \\
\hline Mamá & $75 \%$ \\
\hline
\end{tabular}

En la tabla 8, se puede observar que, bajo la pregunta: ¿Quién compra los recursos tecnológicos que tienes?, los estudiantes respondieron, mayoritariamente, que sus madres son las encargadas de comprar sus recursos tecnológicos (75\%), sobrepasando en cantidad al padre (60\%).

El siquiatra Elías Arab, en su artículo «Recursos tecnológicos y sus múltiples efectos», ${ }^{6}$ habla sobre la influencia de las tecnologías en los niños y la función de los padres frente a esta nueva manera de comunicación, recalcando que hay que considerar que la red es un espacio ilimitado, que no está exento de riesgos; $y$ son finalmente los padres los encargados de la crianza y la supervisión de los hijos. En resumen, siempre es responsabilidad de los adultos, especialmente a edades tempranas.

\section{Aplicaciones más populares}

Tabla 9. Aplicaciones más conocidas por los estudiantes

\begin{tabular}{|c|c|}
\hline Aplicaciones & Porcentaje \\
\hline Facebook & $96 \%$ \\
\hline Youtube & $96 \%$ \\
\hline Wikipedia & $78 \%$ \\
\hline Google Chrome & $89 \%$ \\
\hline Power Point & $87 \%$ \\
\hline
\end{tabular}

$\mathrm{Al}$ analizar los resultados se puede observar que la aplicación tecnológica que los estudiantes más conocen es Facebook, que es una red social muy popular a nivel mundial. Este sitio es un servicio gratuito que permite conectar a las personas en internet. Al ser usuario registrado en su página, se puede gestionar un espacio personal: crear álbumes de fotos, compartir vídeos, escribir notas, crear eventos o compartir el estado de ánimo con otros usuarios de la red. El gran número de usuarios de que dispone, la aceptación que ha tenido, $y$ las facilidades de accesibilidad que ofrece; así como el acceso a la plataforma desde terminales móviles, ha permitido que esta red haya crecido muy rápidamente en poco tiempo.

YouTube $^{7}$ es otra de las aplicaciones más conocidas por los estudiantes, ocupando el mismo porcentaje que Facebook; es decir, son recursos muy populares y de fácil utilización y acceso de cualquier usuario de internet.

6. Extraído de: http://www.educarchile.cl/ech/pro/app/detalle?ID=136523

7. YouTube, programa de alojamiento de videos en Internet. Fue fundado en el año 2005. Este sitio permite a sus usuarios colgar, ver y compartir videos. 
El navegador web Google Chrome se encuentra en el tercer lugar de las aplicaciones más conocidas por los estudiantes, luego sigue el software de productividad Power Point, ${ }^{8}$ el cual es conocido ya que lo utilizan para realizar presentaciones en diversas asignaturas. Según diversos artículos la herramienta más utilizada para presentar información en el aula es Power Point, tanto para profesores como para estudiantes.

Por último, pero no con menos importancia le sigue Wikipedia. El proyecto Wikipedia comenzó el año 2001, y es uno de los diez sitios más visitados en internet, en él se puede encontrar información igual que en las enciclopedias de papel, lo que caracteriza a esta enciclopedia virtual es que los artículos son redactados por voluntarios, solo basta tener una cuenta para poder escribir. Por ello existe cierta controversia en torno a la confiabilidad en el uso de la información disponible, aunque actualmente las normas de control de la información son mucho más rigurosas.

Aplicaciones más utilizadas por los estudiantes

Tabla 10. Aplicaciones más utilizadas por los estudiantes

\begin{tabular}{|c|c|}
\hline Aplicaciones & Porcentaje \\
\hline Facebook & $75 \%$ \\
\hline Youtube & $80 \%$ \\
\hline Wikipedia & $78 \%$ \\
\hline Google Chrome & $78 \%$ \\
\hline Power Point & $73 \%$ \\
\hline
\end{tabular}

Estos resultados no se distancian de los recursos señalados en la tabla anterior, ya que los estudiantes coinciden en que los recursos más conocidos son también los más usados por ellos.

La red social de Facebook presenta una baja, ya que un $96 \%$ de los estudiantes dice conocerla; sin embargo, solo un 75\% la utiliza.

Existen estadísticas sorprendentes sobre el uso de juegos en red, en especial el uso de juegos sociales de Facebook, lo cual se indica como una tendencia creciente en la web, donde se menciona que un $53 \%$ de los usuarios de Facebook juegan juegos; de los cuales el 19\% dice ser adicto; el 20\% de ellos han usado dinero real para beneficios en el juego, mientras que 56 millones de personas juegan diariamente. El 50\% de entradas a Facebook es para jugar. ${ }^{9}$

YouTube mantiene el primer lugar, siendo que para los niños es un espacio de búsqueda constante de videos, vinculando los contenidos más activamente con sus fantasías y deseos. Por tanto puede ser entendido como un espacio de creación y recreación, lo cual podría ser aprovechado por los docentes para aumentar la motivación en el aprendizaje de sus estudiantes.

Mediante ambos resultados, tanto el uso de Facebook como de YouTube, se pueden plantear varias interrogantes, como por ejemplo, la orientación de los docentes hacia sus estu-

8. Extraído de "Qué hacer con la tecnología en el aula: inventario de usos de las TIC para aprender y enseñar". Disponible en: http://www.scielo.org.co/scielo.php?pid=S0123-12942009000200012\&script=sci_arttext

9. xtraído de: http://soxialmedia.com/estadisticas-sorprendentes-sobre-juegos-sociales-de-facebook/ 
diantes para la realización de trabajos, a través de Power Point, o la búsqueda de información por medio de navegadores.

Existen estudios que señalan las consecuencias negativas del acceso a estas nuevas redes sociales: Francisco Bernete (2009) dice que

este fenómeno afecta profundamente a las relaciones entre ellos (estudiantes). En primer lugar, la mensajería instantánea les proporciona el escondite perfecto para expresarse sin exponerse. Pueden decir lo que quieran sin temor a la reacción del otro. Están en el entorno seguro de su pantalla, sin que nadie les vea y sin tener que dar más explicaciones que las que quieran dar. Esto les provoca una pérdida de habilidad en el intercambio personal (la comunicación personal se aprende practicando) y puede desembocar en una especie de "analfabetismo relacional" 10 que les hará el camino mucho más difícil cuando como adultos no tengan más remedio que interactuar con los demás.

Según Del Moral (2005) existen cuatro empleos generales que fomentan el uso de de las redes sociales: Mantenimiento de amistades; nueva creación de amistades; entretenimiento; gestión interna de organizaciones empresariales. Esto lleva a pensar que todos los intercambios y/o formas de comunicación permiten la interacción con personas que sería imposible contactar por otros medios, ya sea por su lejanía física o temporal; surgiendo incluso relaciones de amistad que luego se transforman en relaciones de pareja, entre otras. Estas son solo algunas de las grandes ventajas de las redes sociales en la actualidad.

\section{Utilización de recursos}

Tabla 11. Usos que da el estudiante a los recursos de los que dispone

\begin{tabular}{|c|c|}
\hline Actividad & Porcentaje \\
\hline Descargar música & $91 \%$ \\
\hline Jugar en línea & $75 \%$ \\
\hline $\begin{array}{c}\text { Ver vídeos en } \\
\text { internet (música, } \\
\text { juegos, series, } \\
\text { tutoriales, etc.) }\end{array}$ & $85 \%$ \\
\hline Ver televisión & $89 \%$ \\
\hline Escuchar música & $95 \%$ \\
\hline
\end{tabular}

De una lista de alternativas, los estudiantes marcaron las actividades que realizan y las que no realizan con sus recursos tecnológicos. Como lo muestra la tabla 11, un gran porcentaje de los estudiantes encuestados señala que las actividades que realizan con mayor frecuencia están directamente relacionadas a actividades recreativas y de ocio como ver televisión, ver videos en internet y escuchar música. Esto se confirma con estudios como el de Triidad y Zlachesvsky (2013), que señalan que «el consumo de la televisión se complementa, y a veces compite con el consumo de Internet, más específicamente con el de videos en YouTube», lo que permite que

10. Analfabetismo relacional se entiende como la ausencia de las habilidades sociales básicas. 
los niños vean en internet lo que no pueden ver en la televisión.

Los dos últimos resultados arrojados (ver televisión y escuchar música) se encuentran directamente relacionados con otro analizado anteriormente, en el que se señala que los recursos tecnológicos más presentes en los hogares son la televisión y la radio.

Una revisión realizada por Comstock y Paik (1991) indica que

la relación de los niños con la televisión está siendo determinada por su disponibilidad de tiempo libre, una aproximación no selectiva en su programa elegido y una escasa implicación con lo que ven. Estas pautas pueden cambiar en la medida en que las telecomunicaciones y otros dispositivos multimedia (canales musicales, juegos de ordenador, consolas) están cada vez más dirigidos a los niños y a los jóvenes como nuevos consumidores con poder adquisitivo y que prefieren tener su propio equipamiento antes que compartir el familiar.

Tabla 11. Actividades menos realizadas por los estudiantes

\begin{tabular}{|c|c|}
\hline Actividad & Porcentaje \\
\hline Ver vídeos educativos & $36 \%$ \\
\hline $\begin{array}{c}\text { Comunicarse a través } \\
\text { de correo electrónico }\end{array}$ & $40 \%$ \\
\hline Buscar libros & $45 \%$ \\
\hline
\end{tabular}

La tabla muestra las actividades menos realizadas por los estudiantes, siendo estas ver videos educativos, buscar libros y comunicación vía correo electrónico; esto se puede deber a que los niños utilizan más las redes sociales para la comunicación con sus pares.

\section{Discusión}

De la investigación se evidencian varios antecedentes relacionadas con el acceso y uso de tecnología por parte de los estudiantes de quinto y sexto año de Educación Básica de la Escuela España en Osorno, Chile.

Un gran porcentaje de estudiantes dispone de internet en sus hogares, pese al nivel de vulnerabilidad en que se encuentran clasificados; las familias cuentan actualmente con diversas alternativas a su alcance para contar con este servicio. Esta información se respalda con los resultados de la tercera medición del Índice de Generación Digital (IGD), elaborado anualmente por Educarchile, VTR Banda Ancha y Adimark en Chile, la cual señala que un $62.8 \%$ de los estudiantes tienen computadora en sus hogares. ${ }^{11}$ Junto a lo anterior existen antecedentes de que los hogares chilenos están accediendo a internet, según la Subsecretaría de Telecomunicaciones (Subtel), se registró un notable aumento de los hogares con conexión a este servicio, pasando de $23.9 \%$ en el año de 2009, 53.6\% en el año 2012. ${ }^{12}$

11. Extraído de: http://www.relpe.org/wp-content/uploads/2013/04/04-Uso-Responsable-de-las-TIC.pdf

12. Extraído de: http://desarrollotics.blogspot.com/2012/10/nueve-de-cada-diez-estudiantes-chilenos.html 


\section{Establecimiento y recursos}

En el establecimiento existes múltiples recursos tecnológicos a disposición de los docentes para el trabajo con sus estudiantes, pero pese a la variedad y cantidad estos, sólo mencionan como recursos utilizados para su proceso de enseñanza y aprendizaje: impresora, proyector, fotocopiadora y computadores de escritorio. Junto con esto los estudiantes mencionan ir al laboratorio para mantenerse ocupados cuando no hay docente a cargo del grupo. Es decir no existe - en algunos casos - una planificación intencionada para una integración eficiente de las tecnologías en los procesos de enseñanza y aprendizaje.

Los estudiantes mencionan que los recursos que utilizan en el laboratorio corresponden a software de productividad como el Power Point para realizar presentaciones; sin embargo, no existe un trabajo previo para una búsqueda eficiente de información, por lo cual los estudiantes solo copian y pegan, para luego realizar una exposición. Esta realidad es algo muy frecuente en todos los niveles educativos, según Jorge A. Gálvez en su ensayo «Más allá de copiar y pegar». ${ }^{13}$ Producto de su experiencia, destaca algunos fenómenos repetitivos en torno a esta temática, siendo una conclusión que «en la mayoría de los casos, el estudiante se limita a copiar textos y a pegarlos en su trabajo sin ninguna lectura analítica y reflexiva sobre su pertinencia». Sin duda esto refleja la de muchos estudiantes de Chile y de otros lugares del mundo.

\section{Estudiantes y recursos}

Los recursos que predominan como propios para el estudiante son televisor y radio, seguida por los celulares y computadores (de escritorio y Notebooks). Mencionan además que quienes proveen de estos recursos son los padres - en su mayoría - la madre.

Respecto al conocimiento de aplicaciones tecnológicas las predominantes son Facebook y YouTube. Estos resultados no difieren mucho respecto a las más utilizadas; sin embargo es aquí donde el docente debe prestar atención ya que los estudiantes utilizan en forma muy frecuente la enciclopedia virtual Wikipedia. La cual es catalogada como un espacio en internet en el que no se puede confiar, según Albeiro Rodas en su artículo Wikipedia ¿Es confiable? ${ }^{14} \mathrm{El}$ autor destaca que «Wikipedia no puede ser referencia de ningún estudio, tarea, trabajo de grado, informe empresarial, reporte nacional, fuente informativa para la prensa ni nada de esas cosas...», porque existen opiniones personales, información carente de fuentes autorizadas, información irrelevante, entre otros razones. Pero a la vez existen múltiples investigaciones, como el análisis comparativo realizado por la revista científica Nature, el cual muestra que el margen de error entre publicaciones como la Enciclopedia Británica y Wikipedia no es tan amplio, en lo que a temas científicos concierne; es decir, esta última sería casi tan rigurosa como la prestigiosa Enciclopedia Británica. Esto hace pensar que el docente debe ser el 
agente responsable de dirigir los procesos de búsqueda de información de sus estudiantes, preocupándose de buscar las fuentes de información más confiables.

Llama la atención la poca utilización que se hace del procesador de textos Word, software que permite crear documentos incluyendo fotografías o ilustraciones como imágenes o como fondo, y agregar figuras como mapas y tablas.

\section{Utilización frecuente}

Los usos mayormente dados por los estudiantes corresponden a entretenimiento y se condice con los resultados de las aplicaciones más utilizadas, ya que mayoritariamente ven televisión, ven videos en internet, escuchan música y se conectan a Facebook.
En términos generales, los estudiantes no aprovechan en términos educativos los recursos tecnológicos de que disponen, como podría ser la búsqueda de libros o complementar sus lecturas con fichas bibliográficas encontradas en la web, o búsquedas de información de interés personal.

Pese a que actualmente existe un acceso masivo a las tecnologías por parte de las familias chilenas, no existe un trabajo conjunto para aprovechar las potencialidades que esta nos ofrece en términos educativos, lo cual debiese ser una preocupación permanente de las entidades involucradas.

\section{Referencias}

Bernete, F. (2009). Uso de las TIC. Relaciones sociales y cambios en la socialización de las y los jóvenes. Recuperado de: http://www.injuve.es/sites/default/files/RJ88-08.pdf

Buchi, R. (2002). Internet en Chile. Recuperado de: http://www.dii.uchile.cl/ revista/ArticulosVol5-N2/04-Buchi.pdf

Castells, M. (2001). La era de la información. Vol 1. La Sociedad Red. Segunda edición, p 60, Madrid, España, Alianza Editorial S.A.

Comstock, G; Paik, H. (1991). Television and children; United States.

Hernández, R.; Fernández, C., y Baptista, P. (2010). Metodología de la Investigación. $5^{\circ}$ edición. México: McGraw Hill Interamericana.

Del Moral, J.A (2005). Redes Sociales ¿Moda o nuevo Paradigma? Asociación de usuarios de Internet. Madrid.

MINEDUC (s.f.). Preguntas frecuentes. Recuperado de: http://www.enlaces.cl/tp_enlaces/portales/tpe76eb4809f44/uploadImg/File/Enlaces_FAQ.pdf 
Oliva, A. (2012). La brecha digital en Chile: más allá de la red de acceso. Recuperado de: http:// www.acorn-redecom.org/papers/proceedings2012/009Torres_Espanol.pdf

Prensky, M. (2001). Nativos e inmigrantes digitales. Distribuidora SEK, S.A.

Serrano, A.; Evelio, M. (2003). La brecha digital: mitos y realidades. Universidad Autónoma de Baja California. Departamento Editorial Universitaria. México.

Toffler, A. (1993). La Tercer Ola, Editorial Plaza \& Janes, Estados Unidos

Trinidad, R.; Zlachesvsky, N. (2013). Jugar en contextos tecnológicos: uso y disfrute de internet por niños y niñas de 8 a 10 años en Argentina, Paraguay y Perú. Pontificia Universidad Católica del Perú, Universidad de Buenos Aires, Argentina. Recuperado de: http://www3.ulima.edu.pe/ Revistas/contratexto/13\%20-\%2021.pdf

UNESCO (1992) Boletin Proyecto Principal de Educación en América Latina y el Caribe Santiago, Chile.

UNESCO (2005). Hacia las sociedades del conocimiento. Informe Mundial de la Unesco. Francia UNESCO (2013). Enfoques estratégicos sobre las TICS en educación e América Latina y el Caribe. Recuperado de: http://www.unesco.org/new/fileadmin/MULTIMEDIA/FIELD/Santiago/ images/ticsesp.pdf 